Documentation et bibliothèques

DOCUMENTATION BIBLIOTHËQUES

\title{
Contrôle bibliographique universel dans les pays en développement. Éléments de solutions informatiques
}

\section{Rosario de Varennes}

Volume 20, numéro 1, mars 1974

Automatisation et documentation

URI : https://id.erudit.org/iderudit/1055702ar

DOI : https://doi.org/10.7202/1055702ar

Aller au sommaire du numéro

\section{Éditeur(s)}

Association pour l'avancement des sciences et des techniques de la documentation (ASTED)

\section{ISSN}

0315-2340 (imprimé)

2291-8949 (numérique)

Découvrir la revue

\section{Citer cet article}

de Varennes, R. (1974). Contrôle bibliographique universel dans les pays en développement. Éléments de solutions informatiques. Documentation et bibliothèques, 20(1), 3-7. https://doi.org/10.7202/1055702ar
Résumé de l'article

En vue de faciliter le travail qui s’amorce dans les pays en développement, l'auteur recommande un contrôle bibliographique universel (ISBD, ISBN, ISSN, ISRN, etc.) réalisé grâce à l'informatique. Le format MARC pourrait servir de modèle. Il faudrait améliorer également les moyens d'expression du contenu des documents, c'est-à-dire les répertoires de vedettes-matière, les thesauri, les systèmes de classification, etc. La très grande diversité des langues entraînera des problèmes aigus de traduction et de conversion.
Tous droits réservés (C) Association pour l'avancement des sciences et des techniques de la documentation (ASTED), 1974
Ce document est protégé par la loi sur le droit d'auteur. L'utilisation des services d'Érudit (y compris la reproduction) est assujettie à sa politique d'utilisation que vous pouvez consulter en ligne.

https://apropos.erudit.org/fr/usagers/politique-dutilisation/ 


\section{Contrôle bibliographique universel dans les pays en développement. Éléments de solutions informatiques. *}

Rosario de Varennes

Conseiller à l'analyse et à l'automatisation des services

Bibliothèque de l'Université Laval

Québec

En vue de faciliter le travail qui s'amorce dans les pays en développement, l'auteur recommande un contrôle bibliographique universel (ISBD, ISBN, ISSN, ISRN, etc.) réalisé grâce à l'informatique. Le format MARC pourrait servir de modèle. Il faudrait améliorer égale-

ment les moyens d'expression du contenu des documents, c'est-à-dire les répertoires de vedettes-matière, les thesauri, les systèmes de classification, etc. La très grande diversité des langues entraînera des problèmes aigus de traduction et de conversion.

\section{Préambule}

II est fort possible que l'ambition d'un contrôle bibliographique universel, au sens complet du terme, en dépit des moyens techniques désormais à notre disposition, soit aussi illusoire à la fin de notre vingtième siècle qu'elle l'a été à la fin du dix-neuvième, et peut-être bien que le projet ambitieux de I'UNISIST, dans ses diverses ramifications, s'avérera un rêve doré de l'humanité au même titre que le projet de bibliographie universelle d'Otlet et Fontaine il y a quelque soixantequinze ans.

Toutefois, on peut prendre pour acquis que, de plus en plus, d'ici l'an 2000 , la source privilégiée d'information sur les productions bibliographiques nationales de tout genre, quel qu'en soit le support physique, sera la bibliographie nationale courante du pays d'origine. Ceci en contrepartie de la situation actuelle où certains organismes nationaux d'envergure, par exemple la Bibliothèque du Congrès américain, remplissent le rôle d'agence universelle d'information bibliographique. En raison de la quantité même de la production mondiale, mais aussi à cause de la promotion des services bibliographiques nationaux et des développements de l'informatique et de la téléinformatique, une telle conjoncture bibliographique deviendra de moins en moins supportable et rentable, et, partant, souhaitable.

Sur la base de cette prémisse, il faut donc envisager le moyen le plus efficace d'en arriver

\footnotetext{
* Communication présentée à Grenoble, France, dans le cadre d'une Table ronde de la FIAB sur le contrôle bibliographique universel dans les pays en développement, tenue du 22 au 25 août 1973, à l'intention de bibliothécaires francophones d'Afrique et d'Asie.
}

au contrôle bibliographique universel par le truchement des bibliographies nationales courantes, tant sous l'aspect de la collecte que sous celui de l'échange des données. $F$. Kaltwasser a déjà tracé le plan essentiel à suivre' et Suzanne Honoré l'a précisé dans le cadre de LIBER. ${ }^{2}$ Pour notre part, en songeant à une application plus appropriée à la situation des pays en développement, nous verrions deux niveaux de réalisations susceptibles d'assurer le contrôle bibliographique universel dans ces pays. En fait, il s'agit de politiques d'action à court et à long terme, assorties de recommandations appropriées.

\section{C.B.U. brut}

Dans un premier temps, on ne viserait qu'un objectif minimal de normalisation, assez théorique peut-être, mais constituant une assise solide pour tout développement ultérieur.

En effet, il serait illusoire d'attendre l'uniformisation des règles de catalogage des divers pays ou le lancement officiel d'un formatmachine accepté internationalement avant d'entreprendre l'échange international d'informations bibliographiques manuelles ou ordinolingues. Un effort sérieux de normalisation à l'échelle du globe, tant d'un point de vue bibliographique qu'informatique, amorcé d'une part avec la Conférence internationale

1. Franz Georg Kaltwasser, "Le Contrôle bibliographique universel», Bulletin de l'Unesco à l'intention des bibliothèques, vol. 25, no 5 (septembre-octobre 1971), 268-276

2Suzanne Honoré, "Du catalogage en coopération au contrôle bibliographique universel", Association des bibliothécaires français. Bulletin d'informations, no 77 (4e trimestre 1972), 201-206. 
de Paris sur les principes de catalogage en 1961 et, d'autre part, avec le lancement du projet MARC américain en 1966, permet de fixer dès maintenant des normes minimales acceptables, qu'on ait ou non une bibliographie nationale courante en opération. C'est pourquoi, nous recommandons en premier lieu:

1 - l'acceptation de principe immédiate et la mise en application dans les meilleurs délais, pour la rédaction des notices, des descriptions bibliographiques normalisées internationales déjà élaborées ou en cours, nommément I'ISBD (M), L'ISBD (S), I'ISBD (AV), d'autant plus qu'elles se prêtent aisément, grâce à leur ponctuation normalisée, au traitement par ordinateur.

Nous croyons fermement que si, à brève échéance, la plupart des bibliographies nationales courantes respectaient cette exigence minimale, nous aurions atteint un premier niveau de contrôle bibliographique universel, contrôle, pour ainsi dire, de la donnée brute, déjà suffisant pour établir un réseau mondial efficace d'échange. De plus, nous sommes d'avis que ce niveau d'enregistrement répondrait à la majorité des besoins d'information bibliographique.

Bien sûr, les descriptions bibliographiques, dans leur état actuel, laissent encore beaucoup à désirer. Une réunion internationale pour la révision de l'ISBD (M) se tient justement aujourd'hui et demain, ici même à Grenoble, dans le cadre des activités de la Commission de catalogage de la FIAB. Un point qu'il vaudrait la peine de soulever à propos de cette norme serait l'établissement d'une codification internationale des éléments de description au niveau de la collation et des notes, de façon à en permettre une conversion automatique aux diverses langues nationales. Suzanne Honoré fait une proposition identique quoique moins radicale. ${ }^{3}$

L'on sait que I'ISBD comporte la mention des numérotations internationales de documents. À ce propos, nous faisons la recommandation complémentaire suivante:

2 - la promotion conjointe par les bibliothécaires, les éditeurs et les agences gouvernementales des numérotations internationales (ISBN, ISSN, ISRN, etc.) par le truchement, au départ, d'une représentation officielle au sein de l'AFNIL. ${ }^{4}$

Point n'est besoin d'insister sur les avantages de telles numérotations pour l'édition dans les pays du Tiers-Monde. Énumérons les suivants: meilleur contrôle bibliographique, plus grande facilité d'identification au moment de la commande et enregistrement accéléré dans des banques de données internationales, telles celle de France-Expansion reflétée dans le "Répertoire général des livres de la francophonie",${ }^{5}$ ou celle de la British National Bibliography cumulant les entrées MARC, LC et BNB et les éditant sur ultramicrofiches dans "Books in English». ${ }^{6}$ À ce propos, on peut encore se référer à des applications diverses de I'ISBN, par exemple pour le prêt entre bibliothèques comme dans le système LASER (London and South Eastern Library Region, United Kingdom) ou pour l'établissement de catalogues collectifs nationaux comme dans le cas du S.A. Unicat (South Africa National Union Catalogue of Monographs) paraissant sur microfiches. ${ }^{7} D^{\prime}$ 'autres applications seront recensées dans un article du $\mathrm{Dr}$ K.W. Neubauer, directeur à Berlin de l'Agence internationale pour la numérotation normalisée du livre, article à paraître prochainement dans le Bulletin de l'Unesco à l'intention des bibliothèques.

D'autre part, d'un point de vue informatique, pour éviter la prolifération de structures incompatibles de présentation physique des données qui rendraient onéreuse, sinon impossible, la transmission de ces données par programme d'ordinateur, nous recommandons ce qui suit:

3 - l'établissement, dans une bibliothèque nationale déjà munie d'une capacité informatique adéquate, par exemple celle d'Abidjan, d'une station pilote pour l'étude concrète des formats de communication MARC déjà sur le marché, et l'élaboration, à partir de ces modèles, d'un format MARC adapté aux besoins bibliographiques des pays en développement.

4. Suzanne Honoré, "L'agence francophone pour la numérotation internationale du livre (A.F.N.I.L.)». Bulletin des bibliothèques de France, $18 \mathrm{e}$ année, no 3 (mars 1973), 87-99.

5. "France Expansion et le Québec". Vient de paraitre. Bulletin du livre au Canada français, vol. 9, no 2 (juin 1973), 16-17

6. J.E. Linford, "Books in English». Canadian Library Journal, vol. 30, no. 2 (March-April 1973), 132-138.

7. «International Standard Book Numbers: Applications...». International Cataloguing, vol. 2, no. 2 (AprilJune 1973), 4-5 
II conviendrait que soient retenues, entre autres, les dispositions du MARC canadien relatives au traitement des termes équivalents en deux langues (en fait, deux ou plusieurs langues) au niveau, particulièrement, des vedettes-auteur et des vedettes-matière MARCAL (MARC para América latina), dans le contexte sud-américain, se prévaudra de cette capacité pour le traitement des publications en espagnol et en portugais. ${ }^{8} / 1$ conviendrait également d'envisager sérieusement, au niveau de cette expérience pilote, l'élaboration d'un programme de recherche documentaire approprié, en s'inspirant de systèmes opérationnels comme SELDOM (Selective Dissemination of MARC) pratiqué à la $\mathrm{Bi}$ bliothèque nationale du Canada. ${ }^{9.10}$ Nous sommes en effet convaincus que, de plus en plus, les outils traditionnels de référence que sont les catalogues sur fiches ou sous forme de volumes céderont le pas à des systèmes de distribution sélective d'informations spécifiques élaborés spécialement pour leurs destinataires sur le support le plus convenable à cette diffusion. Enfin, pour assurer une audience internationale aux travaux de cette cellule pilote et, en contrepartie, pour lui permettre de suivre les développements internationaux sur ce point, nous faisons la recommandation supplémentaire suivante:

4 - qu'au moins à titre d'observateur, le groupe participe aux délibérations du Groupe de travail de la FIAB sur la codification des indicateurs de contenu et à celles des sous-comités appropriés du Comité technique ISO «Documentation» (ISO / TC / 46).

\section{C.B.U. "proprie»}

Jusqu'ici, on aura pu réussir à cerner étroitement l'enveloppe externe du document et à en refléter les informations de base dans des cadres normalisés de présentation qui en permettent la manipulation en ordinateur et la "re-présentation" sous forme de produits divers déjà fort appréciables pour le C.B.U.

8. "Projets coopératifs et un format MARC pour l'Amérique latine" [LILIBU - CATACEN - MARCAL], ACcessible, Nouvelles, Direction de la recherche et de la planification, Bibliothèque nationale, Ottawa, Canada, vol. I, no 4 (juin 1973), 13-14

9. Georg R. Mauerhoff et Richard G. Smith, "A MARC 11 - Based program for retrieval and dissemination". Journal of Library Automation, vol. 4, no. 3 (September 1971), 141-158.

10. Lorne R. Buhr, "Selective dissemination of MARC a user evaluation". Journal of Library Automation, vol 5, no. 1 (March 1972), 39-50.
Dans un deuxième temps, il faudrait s'attarder à des préoccupations beaucoup plus essentielles concernant d'abord les concepts capables d'exprimer, si possible, la quintessence du contenu - qu'il s'agisse de termes séparés, structurés ou non (vedettes-matière, descripteurs, unitermes) de chaînes de concepts post- ou pré-coordonnés ou de condensés analytiques - concernant aussi la corrélation de ces concepts à des schèmes classificatoires appropriés et, enfin, leur expression correcte dans les diverses langues de traitement, sans compter les complexités des règles de catalogage comportant également une incidence linguistique. Donc, problèmes de répertoires de vedettes-matière, de thesauri, de tables de classification, de normes catalographiques, doublés de problèmes linguistiques cruciaux.

Nous n'avons nullement l'intention d'épiloguer sur ces questions; tout au plus nous permettrons-nous d'émettre quelques suggestions d'ordre pratique et de vous référer à deux dossiers de travail que nous avons produits en 1970 et 1971 sur ce sujet ${ }^{11.12}$.

Auparavant, il paraît opportun d'insister sur la nécessité d'apporter la meilleure solution possible au problème difficile d'équivalence entre langues à l'intérieur des systèmes documentaires automatisés. Nous ne saurions mieux faire à ce propos que de vous recommander la lecture attentive de l'article de Jean Lochard, ingénieur en chef à l'AFNOR, paru en 1971 dans le Bulletin de l'Unesco à l'intention des bibliothèques ${ }^{13}$. II reste à souhaiter que des organismes comme l'Unesco s'en inspirent dans la mise en œuvre de systèmes d'envergure tel I'UNISIST.

11. Rosario de Varennes, "Normalisation de la classification et des vedettes-matière». Conférence nationale sur la normalisation du catalogage. Bibliothèque nationale du Canada, 19-20 mai 1970, Document de la conférence, no 5, $11 \mathrm{p}$. Aussi classée dans le système américain ERIC sous ERIC-CLIS ED 058901

12. "Quelques exigences concernant le contenu bilingue d'un service "MARC " canadien éventuel et recommandations à ce propos", préparé par le 2e sous-groupe de travail: Rosario de Varennes, président, Marcel Fontaine, Jean Lunn", Bibliothèque nationale du Canada, Groupe de travail sur MARC, MARC CANADIEN Rapport d'activités et recommandations du Groupe de travail sur MARC concernant un Format MARC canadien pour les monographies et un Format MARC canadien pour les publications en série. Ottawa, Bibliothèque nationale du Canada, 1972. Annexe C, 232-241

13. Jean Lochard, "Automatisation de la documentation et normalisation", Bulletin de l'Unesco à l'intention des bibliothèques, vol. 25, no 3 (mal-juin 1971), 153 . 161 
Pour en venir aux suggestions pratiques concernant ce deuxième niveau, plus en profondeur, du contrôle bibliographique universel, il y aurait d'abord l'accélération de la production des listes d'autorités de patronymes. C'est certainement un domaine où nos collègues bibliothécaires des pays en développement peuvent rendre un service éminent à la communauté bibliographique internationale. Ces listes compléteraient avantageusement les répertoires de collectivités-auteurs et d'auteurs classiques anonymes en préparation à la FIAB.

Il se pose ensuite la question de l'accès par sujet au contenu de la documentation. Au Canada présentement, on s'apprête à établir l'équivalence automatique de l'anglais au français, et vice versa, des termes du répertoire de Subject Headings Used in the Dictionary Catalogs of the Library of Congress ${ }^{14}$ et du Répertoire de vedettes-matière de la $\mathrm{Bi}$ bliothèque de l'Université Laval, ${ }^{15}$ qui sont acceptés comme normes nationales ${ }^{16}$. On pourrait élargir l'expérience à la "Lista de Encabezamientos de Materia para Bibliotecas" de l'Unión Panamericana ${ }^{17}$ et concevoir finalement une codification numérique des termes avec leurs équivalents en plusieurs langues capables d'être produits automatiquement. On pourrait essayer encore de faire le lien entre cette recherche et celle qui est effectuée par le nouveau Groupe de travail de la FID sur un code de référence-sujet (FID / SRC «Subject-field Reference Code»). Toutefois, devant la remise en question, ces dernières années, de la valeur documentaire de cette approche-sujet traditionnelle, nous suggérons plutôt un essai d'application bilingue, avec le français et une langue vernaculaire africaine ou asiatique, du système PRECIS (PREserved Contact Index System) ${ }^{18}$ de la BNB susceptible de déboucher sur un thesaurus universel multilingue. Au Canada, une expérience similaire est en cours avec l'anglais et le français au College Bibliocentre de Toronto, grâce à un octroi de la Bibliothèque nationale. Par ail-

14. Library of Congress, Subject Cataloguing Division, Subject Headings used in the Dictionary Catalogs of the Library of Congress, 7th ed., Washington, D.C., 1966 et suppléments.

15. Université Laval, Québec (Canada), Bibliothèque, Répertoire de vedettes-matière, 7e éd., Québec, 1972

16. Bibliothèque nationale du Canada, Ottawa, Cataloguing Standards, The Report of the Canadian Task Group on Cataloguing Standards, Ottawa, National Library of Canada, 1972. Recommandations 22 et suivantes.

17. Carmen Rovira et Jorge Aguayo, comp., Lista de Encabezamientos de Materia para Bibliotecas, Washington, Union Panamericana, 1967. 3 vol. (Manuales del bibliotecario, 6). Épuisé leurs, lors d'un exposé devant le Groupe canadien de travail sur MARC portant sur ce système d'indexation monoconceptuelle précoordonnée, l'inventeur de PRECIS, Derek Austin, révéla qu'en cours d'essai une expérience anglais-swahili s'était avérée concluante. À retenir également que PRECIS comporte une codification-machine permettant la génération automatique d'index-sujets avec rotation des termes de la chaîne de descripteurs de chaque analyse.

Concernant les règles de catalogage, serait-il illusoire de suggérer que, d'ici la parution d'une deuxième édition des Anglo-American Cataloging Rules ${ }^{19}$, un groupe de travail international "ad hoc» examine sérieusement la possibilité d'en faire un code international de catalogage. En effet, depuis la première édition de 1967,1$)$ les divergences majeures entre les éditions américaine et britannique ont été éliminées, spécialement depuis l'abandon par la Library of Congress des règles d'exception 98 et 99 (politique de «superposition»); 2) le code a été adapté, à toutes fins pratiques, pour tenir compte des exigences de la description bibliographique normalisée internationale (ISBD) - vous êtes sans doute au courant que la nouvelle rédaction du chapitre 6 sera acceptée officiellement après les délibérations de Grenoble cette semaine; 3 ) le code est déjà ouvert aux règles particulières de traitement pour le non-textuel et la deuxième édition incorporera les dernières normes développées entre-temps à ce sujet ${ }^{20}$. $\left.{ }^{21} ; 4\right)$ enfin une version française des Règles anglo-américaines de catalogage vient de sortir des presses à Montréal, par les soins

18. Derek Austin et Peter Butcher, PRECIS: A Rotated Subject Index System, London, Council of the BNB, 1969. 87p. (SBN 900220-12-0).

- Ibid. Supplément, s.I.n.d., 18p

- Derek Austin. The PRECIS System for Computer

- Generated Indexes and its USE in the BNB, s.I.n.d., $18 p$.

- Derek Austin, "PRECIS Indexing", Information Scientist. vol. 5, no. 3 (Septembre 1971), 95-114.

- Derek Austin, "Classification and Subject Indexing at the British National Bibliography" Canadian Library Journal, vol. 30, no. 2 (March-April 1973), 122-130. - Derek Austin, PRECIS Manual of Indexing, 19721973, Manuscrit.

19. Anglo-American Cataloguing Rules, prepared by the American Library Association, the Library of Congress, the Library Association and the Canadian Library Association, North American Text, Chicago, American Library Association, 1967. (Idem, British text. London. Library Association, 1967).

20. J.R. Weins, S. Lewis et J. Macdonald, Nonbook Materials. The organization of integrated collections, 1st ed., Ottawa, Canadian Library Association, 1973. 107p. (ISBN 088802091 0) 
de l'ACBLF ${ }^{22}$, ce qui assure désormais au code une audience internationale élargie. Ne conviendrait-il pas de faire un pas de plus?

Un dernier point à examiner, les schèmes classificatoires. Devant la complexité et la variété des systèmes utilisés, comportant chacun leurs avantages et leurs limitations, nous n'avons rien de particulier à suggérer sauf de suivre attentivement le développement des travaux du groupe de travail de la FID susmentionné, visant à l'élaboration d'un schème général de classement universel par grandes catégories de sujets, et adapté aussi bien au systèmes manuels qu'informatisés, en vue de l'interconnection et de la coopération entre les systèmes documentaires envisagées par I'UNISIST.

\section{Conclusion}

Lors des assises annuelles de l'ACBLF à Québec, en novembre 1972, nous concluions un exposé sur la téléinformatique en l'an 2000 "par le souhait que l'accès universel à l'information et à l'éducation, grandement soutenu par les possibilités de la téléinformatique, permette de combler le fossé des différences économiques et culturelles entre pays favorisés et moins favorisés et de réaliser enfin le rêve si longtemps caressé par les meilleurs esprits du monde d'un rapprochement réel entre l'Est et l'Ouest" ${ }^{23}$.

Nous aimerions terminer notre entretien d'aujourd'hui dans le même esprit par le souhait que le contrôle bibliographique universel en pays développés ou en développement, et l'interconnection des systèmes documentaires par le truchement de la téléinformatique, grâce à la dimension de la collaboration humaine impliquée à divers niveaux pour leur réussite concrète, contribuent en définitive à faciliter la compréhension mutuelle entre ces deux blocs culturels.

21. Library Association, London. Media Cataloguing rules committee, Nonbook Materials Cataloguing rules. Sous-Presse

22. Paule Rolland-Thomas, Règles de catalogage anglo-américaines. Montréal, Association canadienne des bibliothécaires de langue française, 1973. 424p.

23. Rosario de Varennes, "Chaque citoyen son câble, ou prospective sur la téléinformatique en l'an 2000", Le livre, centre de la documentation, accessible à tous. Rapport des travaux du 28 e congrès de l'ACBLF tenu à Québec, du 1er au 5 novembre 1972, Montréal, 1973, $71-79$

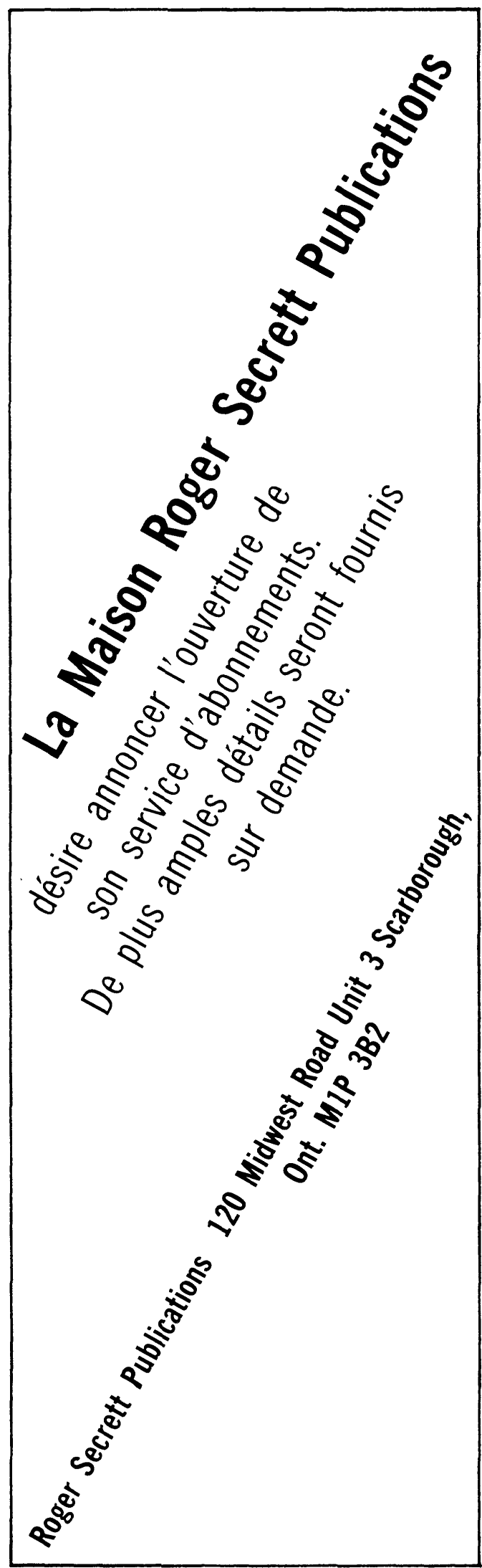

